\title{
New analytical solutions to the nonlinear Boussinesq equation for groundwater flow
}

\author{
Tomás L. Chor, \\ Programa de Pós-Graduação em Engenharia Ambiental, UFPR, \\ 81530-900, Curitiba, PR \\ E-mail: tomaschor@gmail.com, \\ Nelson L. Dias \\ Departamento de Engenharia Ambiental, UFPR, \\ 81530-900, Curitiba, PR \\ E-mail: nldias@ufpr.br, \\ Ailín Ruiz de Zárate \\ Departamento de Matemática, UFPR, \\ 81530-900, Curitiba, PR \\ E-mail: ailin@ufpr.br.

\begin{abstract}
In this communication we briefly describe some results related to the nonlinear differential Boussinesq equation for groundwater flow with an emphasis in hydrology. We review important characteristics of the equation and some of its solutions. The equation is then solved for an infinite aquifer with constant boundary conditions in three distinct ways: one looking for a solution as a function of the similarity variable, that is, the classical approach, and the other two by means of alternative interpretations of variable dependence. All of the solutions are based on series expansions.
\end{abstract}

Keywords: Boussinesq equation, Nonlinear Equations, Hydrology

\section{Introduction}

The Boussinesq equation, first introduced by Boussinesq (1904), models groundwater flow when the effects of capilarity are not important. Mathematically, it can be classified as a nonlinear diffusion equation, with the diffusion coefficient varying linearly. For a homogeneous and horizontal domain, the equation can be written as

$$
n_{e} \frac{\partial h}{\partial t}=k_{0} \frac{\partial}{\partial x}\left(h \frac{\partial h}{\partial x}\right)
$$

where $k_{0}$ and $n_{e}$ are the saturated hydraulic conductivity and the drainable porosity, respectively; $x$ and $t$ are horizontal coordinate and time; and $h(x, t)$ is the water level above the impervious bottom.

One of its most common uses in hydrology is the modelling of aquifer-stream systems, such as the one presented in Fig. 1, which depicts two rectangular banks with a water stream in the middle - a simple representation of a water basin. The Boussinesq equation can be used to solve one of the sides and then extrapolate the solution to the other side by symmetry. With this procedure not only the water table height is known, but also the water discharge at the stream (Brutsaert and Nieber, 1977; Rupp and Selker, 2006). 
The boundary conditions for each side are

$$
h(x, t=0)=H, \quad h(x=\infty, t)=H, \quad h(x=0, t)=H_{0}, \quad x \geq 0,
$$

where it is assumed that the aquifer length $B$ is infinite, so that one can transform (1) in an ordinary differential equation using the well-known Boltzmann transformation of variables

$$
\phi(\xi) \equiv \frac{h(x, t)}{H}, \quad \xi \equiv \frac{x}{\sqrt{4 D t}},
$$

where $D=H k_{0} / n_{e}$. This reduces Eq. (1) and the boundary conditions (2) to

$$
\frac{d}{d \xi}\left(\phi \frac{d \phi}{d \xi}\right)+2 \xi \frac{d \phi}{d \xi}=0
$$

with

$$
\phi(0)=\frac{H_{0}}{H}=\phi_{0}, \quad \phi(\infty)=1, \quad \phi_{0} \geq 0
$$

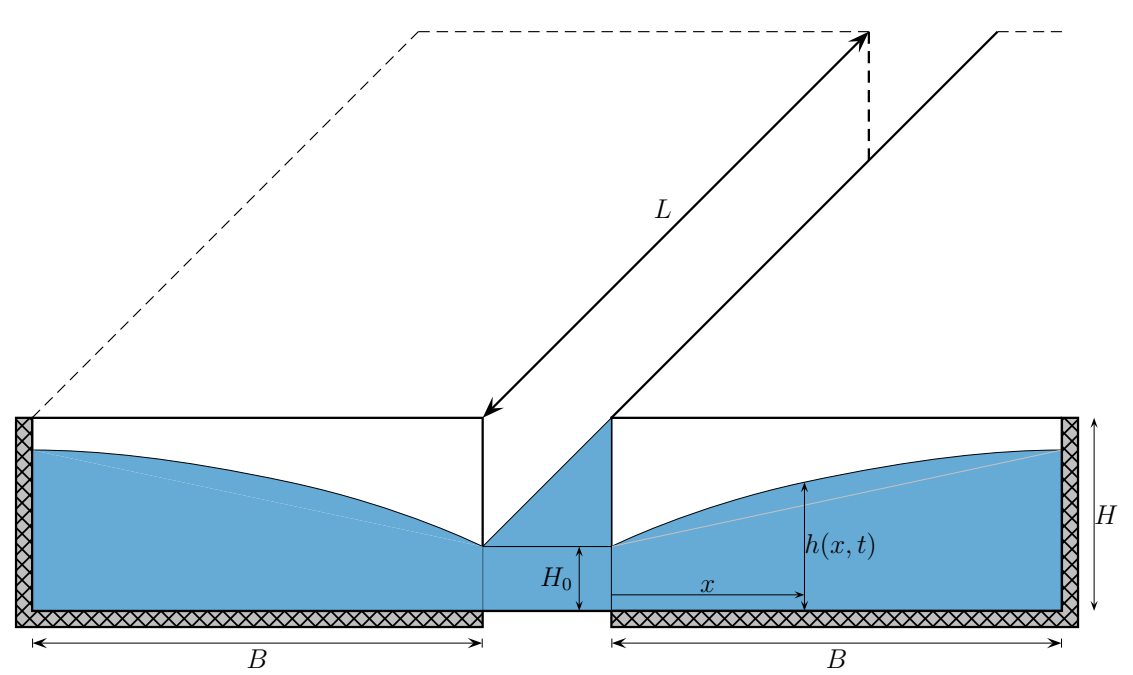

Figure 1: Aquifer-stream system which can be modelled by the Boussinesq equation.

In this work we focus on the ordinary differential equation problem composed by Eqs. (4) and (5), which is valid for early times of a discharging aquifer (Brutsaert, 2005; Chor et al., 2013).

\section{General solution}

In this section we derive a series solution by means of a recurrence relation. The relation derived here generalizes the solutions presented in Chor et al. (2013) for a homogeneous case $\left(H_{0}=0\right)$ and Dias et al. (2014) for a nonhomogeneous case $\left(H_{0} \neq 0\right)$. Begin by integrating Eq. (4) from an arbitrary point $\xi_{a}$ to $\xi$, which produces:

$$
\phi \frac{d \phi}{d \xi}-\psi_{a}+2\left(\left.\xi \phi\right|_{\xi_{a}} ^{\xi}-\int_{\xi_{a}}^{\xi} \phi d \xi\right)=0
$$

where

$$
\psi \equiv \phi \frac{d \phi}{d \xi}
$$

and where we use the notation $\xi_{a}$ for the point of expansion of the series, $\phi_{a}=\phi\left(\xi_{a}\right)$ and $\psi_{a}=\psi\left(\xi_{a}\right)$. 
Assuming that the Boussinesq function $\phi$ can be expressed as

$$
\phi(\xi)=\sum_{n=0}^{\infty} a_{n}\left(\xi-\xi_{a}\right)^{n / 2},
$$

it follows, after substituting Eq. (8) into (6), that

$$
\begin{aligned}
a_{1} a_{0} & =a_{1} \phi_{a}=0, \\
\frac{a_{1}^{2}}{2}+a_{2} a_{0} & =\psi_{a}, \\
3\left(a_{1} a_{2}+a_{3} a_{0}\right)+4 \xi_{a} a_{1} & =0 .
\end{aligned}
$$

The final recurrence relation depends on the conditions at the point of expansion. If $\phi_{a} \neq 0$ (nonhomogeneous case), then $a_{0} \neq 0$ and we have

$$
a_{n+4}=-\frac{1}{(n+4) a_{0}}\left(\frac{n+4}{2} \sum_{k=1}^{n+3} a_{k} a_{n-k+4}+\frac{4 n}{n+2} a_{n}+4 \xi_{a} a_{n+2}\right) .
$$

If, however, $\phi_{a}=0$ (homogeneous case), then $a_{0}=0$ and one has the recurrence relation

$$
a_{n+3}=-\frac{1}{(n+4) a_{1}}\left(\frac{n+4}{2} \sum_{k=2}^{n+2} a_{k} a_{n-k+4}+\frac{4 n}{n+2} a_{n}+4 \xi_{a} a_{n+2}\right) .
$$

It becomes clear after analysing Eqs. (9)-(11) and (8) that if the series is expanded about a point such that $\phi_{a}=0$, then $a_{0}=0$. In this case the first non-zero term of the expansion is $a_{1}=\sqrt{2 \psi_{a}}$, configuring a singular behavior of the series at $\xi_{a}$. If, however, $\phi_{a} \neq 0$, then all the odd numbered coefficients $\left(a_{1}, a_{3}, a_{5} \ldots\right)$ are zero, cancelling out all the fractional exponents and effectively making the series a Taylor series.

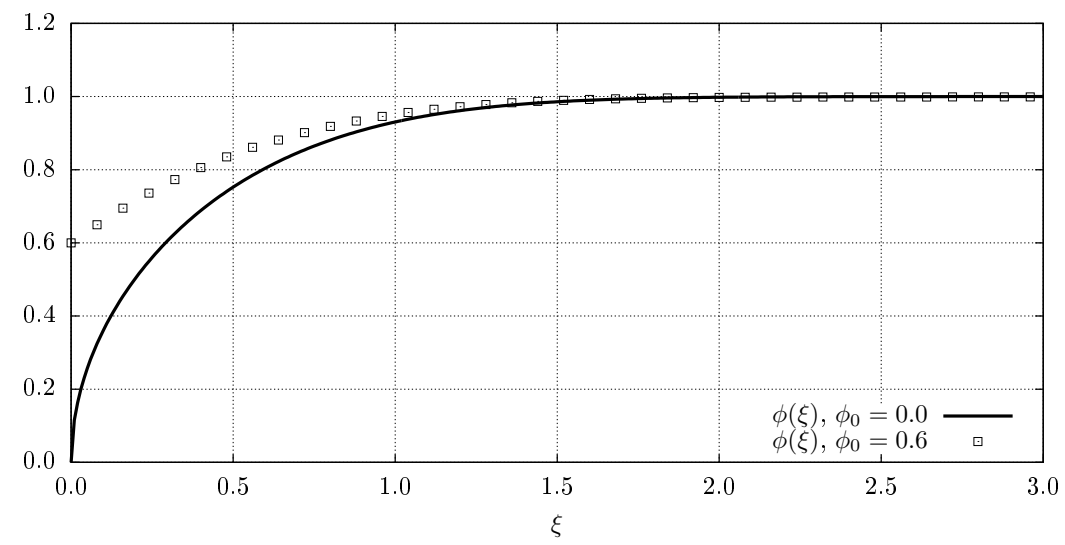

Figure 2: Functions $\phi$ and $\psi$ for the homogeneous case obtained with the recurrence relations given by Eq. (12) (for $\phi_{0}=0.6$ in the dotted line) and Eq. (12) (for $\phi_{0}=0$ in the solid line).

Figure 2 shows the series expansion for $\phi_{0}=0.6$ (dotted line) and for the homogeneous case $\phi_{0}=0$ (solid line). The main advantage of this solution is that it is general enough to allow the use of the principle of analytical continuation in order to reproduce the function in the whole semi-infinite domain with arbitrary precision. This can be done because of the capacity of the series to be expanded about any point in the domain. As an example, the graphs in Fig. 2 were plotted with the help of two successive series expansions, about 0 and 2.3.

The analytical continuation is a necessity, because regardless of the point of expansion the series will not converge in the whole domain. The reason for it lies in the many singularities that appear in the complex-plane of the function, as can be seen in Fig. 3. 

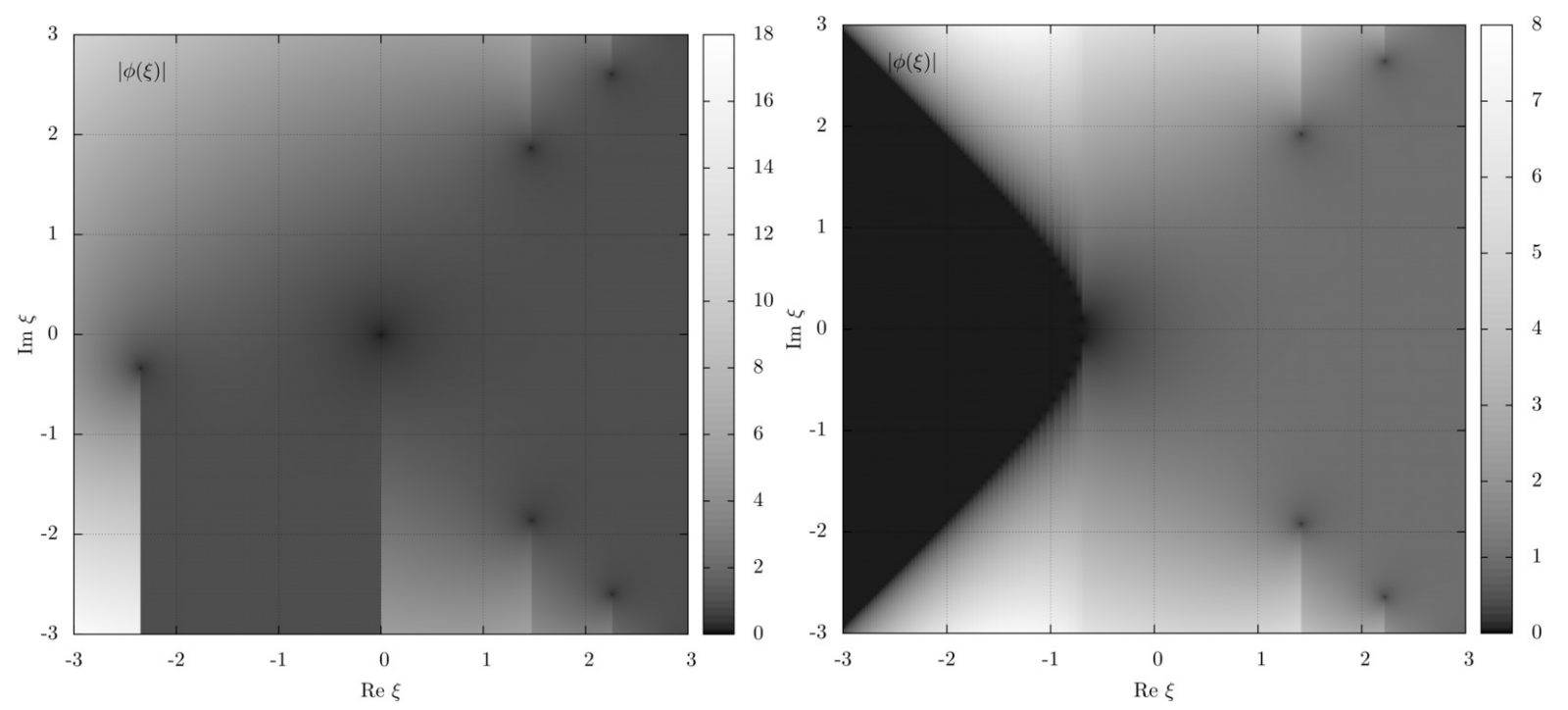

Figure 3: The complex-plane of $\phi(\xi)$, where one can see many singularities represented by the black dots. Left graph is case $\phi_{0}=0$ and graph on the right is case $\phi_{0}=0.6$.

\section{Derivation of an "inverted" series solution}

In this section we tackle the same problem, but with $\xi$ as a function of $\phi$; that is to say we find $\xi(\phi)$. From now on we consider solely the problem expanded about $\xi=0$ (i.e., the variables $\phi_{0}$ and $\psi_{0}$ ). One can easily transform Eq. (4) into

$$
\frac{d}{d \phi}\left(\frac{\phi}{\xi^{\prime}}\right)+2 \xi=0
$$

First assume that $\xi(\phi)$ can be written as

$$
\xi(\phi)=\sum_{n=1}^{\infty} c_{n}\left(\phi-\phi_{0}\right)^{n} .
$$

Consider now Eq. (14) integrated from $\phi_{0}$ to $\phi$ and multiplied by a factor $\xi^{\prime}$ :

$$
\phi-\xi^{\prime} \psi_{0}+2 \xi^{\prime} \int_{\phi_{0}}^{\phi} \xi(\phi) d \phi=0
$$

where the value of $\psi_{0}=\psi(0)$ is necessary to complete the initial conditions of the problem. In Section 4 we will show how to obtain it a priori.

Substituting Eq. (15) into (16) one has

$$
\begin{aligned}
c_{1} & =\frac{\phi_{0}}{\psi_{0}} \\
c_{2} & =\frac{1}{2 \psi_{0}},
\end{aligned}
$$

and a recurrence relation can be derived:

$$
c_{n+2}=\frac{2}{(n+2) \psi_{0}} \sum_{k=1}^{n} \frac{n-k+1}{k+1} c_{k} c_{n-k+1} .
$$

The series solution obtained via equations (15) and (19) is presented in Fig. 4 for $\xi(\phi)$. Figure 4-a shows the direct result of the series (i.e. $\xi(\phi))$ and Fig. 4-b shows the same results inverted back to the regular physical variables and interpolated (i.e. $\phi(\xi))$. 


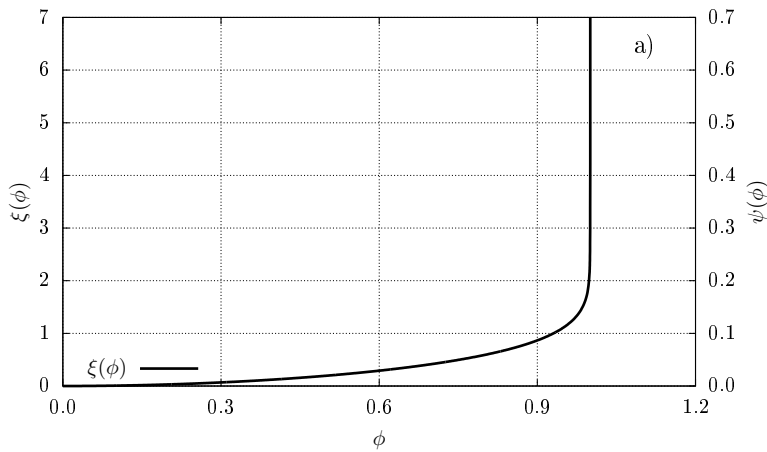

Figure 4: $\xi(\phi)$ obtained via Eqs. (15) and (19) (left) and $\phi(\xi)$ obtained after inverting the results of the same equations (right).

The main advantages of this series solution in comparison to the one given by Eqs. (12)-(13) are: (i) this solution is capable of reproducing the function $\phi$ in the range of interest; (ii) the fact that cases where the point of expansion is a singularity in the $\phi(\xi)$ form do not have to be dealt with differently than an expansion around a non-singular point, i.e. there is only one recurrence relation.

On the other hand, the shortcomings of Eq. (19) with (15) are (i) the need to interpolate the results and trace them back to the form of $\phi(\xi)$ in order for the solution to have real physical application; and (ii) the fact that the convergence of the series close to the pole in $\phi=1$ is slow, which makes necessary the use of a large number of terms in that region.

One feature of Eq. (14) is that it can be used to find the singularities of Eq. (4) (as shown in Fig. 3). This is done by doing a complex integration of Eq. (14) up to the origin $(\phi=0)$. The positions of the black dots in Fig. 3 are the different values that $\xi(\phi)$ takes for different paths of integration made in order to retrieve this variable.

\section{Determination of the initial conditions}

We now focus on the determination of the initial conditions for the problem without the aid of numerical integration, which roughly means knowing the value of the variable $\phi_{0}$ before solving for $\phi(\xi)$ (regardless of the method used to solve). Eq. (4) can be rewritten as

$$
\psi \frac{d^{2} \psi}{d \phi^{2}}=-2 \phi
$$

which has the advantage of eliminating $\xi$ and treating $\phi$ as the independent variable, thus reducing the problem from a semi-infinite domain to a finite one $\left(\phi_{0} \leq \phi \leq 1\right)$. The inversion of varibles produces the conditions

$$
\frac{d \psi}{d \phi}\left(\phi_{0}\right)=0, \quad \psi(1)=0, \quad \psi\left(\phi_{0}\right)=\psi_{0} .
$$

Let us assume then that $\psi(\phi)$ can be expressed as

$$
\psi=\sum_{n=0}^{\infty} b_{n}\left(\phi-\phi_{0}\right)^{n} .
$$




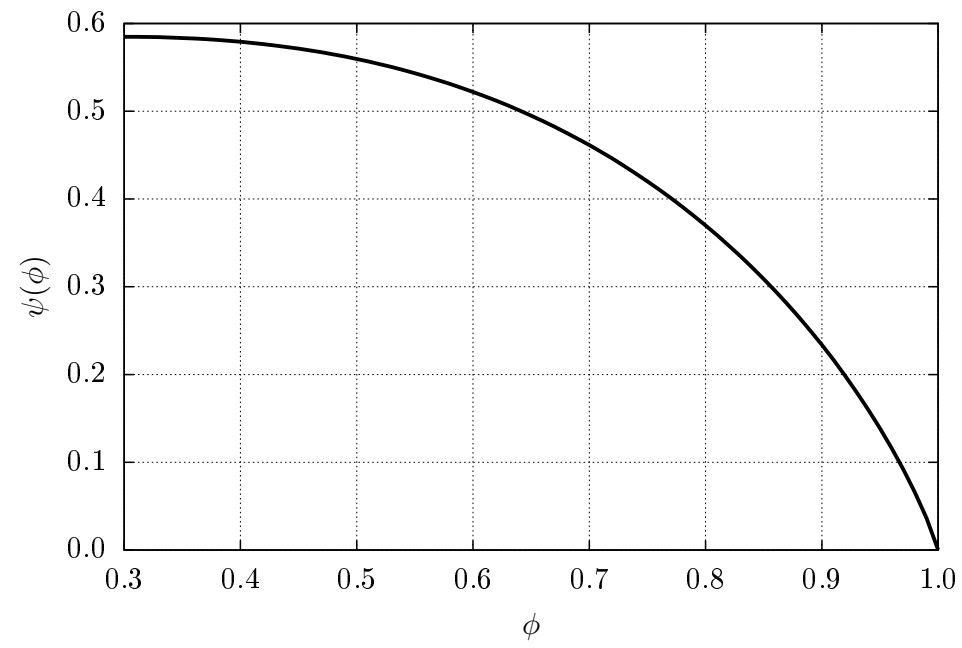

Figure 5: Function $\psi(\phi)$ calculated with the series (22) for $\phi_{0}=0.3$. The value of $\psi_{0}$ was calculated with the new iterative procedure involving Eq. (28).

Substituting Eq. (22) in Eq. (20) along with the boundary conditions, one can derive

$$
\begin{aligned}
& b_{0}=\psi_{0} \\
& b_{1}=0 \\
& b_{2}=-\frac{\phi_{0}}{\psi_{0}} \\
& b_{3}=-\frac{1}{3 \psi_{0}}
\end{aligned}
$$

which makes it possible to write the recurrence relation for $b_{n}$ as

$$
b_{n+2}=-\frac{1}{\psi_{0}(n+2)(n+1)} \sum_{k=1}^{n}(n-k+2)(n-k+1) b_{k} b_{n-k+2}, \quad n>1 .
$$

Fig. 5 illustrates $\psi(\phi)$ obtained with an application of Eq. (27) for the case $\phi_{0}=0.3$. Callegari and Friedman (1968) proved that Eq. (22) with (27) converges for $0 \leq \phi_{0} \leq 1$, so it can be used to reproduce the boundary condition $\psi(\phi=1)=0$. By doing this, one can estimate the correct value of $\psi_{0}$ analytically by solving

$$
\sum_{n=0}^{\infty} b_{n}\left(1-\phi_{0}\right)^{n}=0
$$

As far as the authors are concerned, this determination of $\psi_{0}$ is the first analytical method to obtain the value of $\psi_{0}$ with arbitrary precision for every case within $0 \leq \phi_{0} \leq 1$. The implementation of this method (defined by Eqs. (28) and (23)-(27)) has to be done iteratively since for every new iteration the new $b_{n}$ coefficients have to be recalculated.

\section{Conclusion}

In this work we studied the Boussinesq equation for groundwater flow. Although hydrology applications are our main interest, the results presented here apply to similar nonlinear diffusion equations in which the diffusion coefficient varies linearly with the dependent variable.

We have solved the problem with three methods: two of them effectively produce the desired water table height as a function of the physical variables (i.e., $h(x, t))$, while the third method 
is used primarily to obtain the initial conditions to correctly solve the original boundary value problem.

In practice, with the aid of the solutions presented here, the Boussinesq equation can be solved in a much faster way than by successive numerical integrations in order to find the correct initial condition that match the boundary condition at infinity. Furthermore, with a deeper understanding of this nonlinear diffusion equation, the solution could become a reference for more complicated and more general models.

\section{References}

J. Boussinesq. Recherches théoriques sur l'écoulement des nappes d'eau infiltrées dans le sol et sur le débit des sources. J. Math. Pures Appl., 10:5-78, 1904. 5me Sér.

W. Brutsaert. Hydrology: An Introduction. Cambridge University Press, 2005. ISBN 9780521531863 .

W. Brutsaert and J. L. Nieber. Regionalized Drought Flow Hydrographs From a Mature Glaciated Plateau. Water Resour. Res., 13(3):637-643, June 1977.

A. J. Callegari and M. B. Friedman. An Analytical Solution of a Nonlinear, Singular Boundary Value Problem in the Theory of Viscous Fluids. J. Math. Anal. Appl., 21(1):510-529, 1968.

T. Chor, N. L. Dias, and A. R. de Zárate. An exact series and improved numerical and approximate solutions for the boussinesq equation. Water Resour. Res., 49(11):7380-7387, 2013. doi: $10.1002 /$ wrcr.20543.

N. L. Dias, T. L. Chor, and A. Ruiz de Zárate. A semianalytical solution for the boussinesq equation with nonhomogeneous constant boundary conditions. Water Resources Research, 50 (8):6549-6556, 2014. ISSN 1944-7973. doi: 10.1002/2014WR015437.

D. E. Rupp and J. S. Selker. On the use of the Boussinesq equation for interpreting recession hydrographs from sloping aquifers. Water Resour. Res., 42(12), DEC 28 2006. ISSN 00431397. 